\title{
Chondroprotective efficiencies of intra-articular treatment of glucosamine sulfate and hyaluronic acid
}

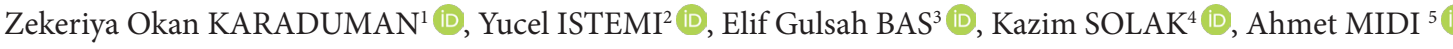 \\ 1 Department of Orthopedics and Traumatology, School of Medicine, Duzce University, Duzce, Turkey \\ 2 Department of Orthopedics and Traumatology, Fatih Sultan Mehmet Training and Research Hospital, Istanbul, Turkey \\ 3 Medical Student, School of Medicine, Bahcesehir University, Istanbul, Turkey \\ 4 Orthopedics and Traumatology Clinic, Duzce Ataturk State Hospital, Duzce, Turkey \\ 5 Department of Pathology, School of Medicine, Bahcesehir University, Istanbul, Turkey \\ Corresponding Author: Ahmet MIDI \\ E-mail: ahmetmidi@yahoo.com
}

Submitted: 11.05.2019 Accepted: 27.07.2019

\begin{abstract}
Objectives: The main purpose of this study was to determine a comparison between the chondroprotective efficacy of intra-articular administrations of glucosamine sulphate (GS) and hyaluronic acid (HA) in the experimental knee osteoarthritis model in rats. Materials and Methods: Forty-five rats were assigned to the following three groups: GS group (Group 1), HA group (Group 2), control group (Group 3). The knee joints of the rats were seen macroscopically and the anterior cruciate ligaments were resected. Two weeks after surgery, an intra-articular injection was applied to each group for three weeks with 1 week interval. In the osteoarthritic rat knee joints, the histopathological effects of tissue regeneration of GS and HA applications were compared with the control group.

Results: The total Mankin Scale was $2.64 \pm 2.56,3.58 \pm 3.9,8.12 \pm 2.80$ for the GS group, HA group and control group respectively. According to these results, the GS and HA were superior to the control group. The values of the total Mankin Scale, cartilage structure, cellular abnormality, matrix staining and tidemark integrity of the GS group were lower than the values of the HA group but this difference was not statistically significant.

Conclusion: According to the results of this study, intra-articular administration of GS for the management of osteoarthritis may be beneficial for patients with knee osteoarthritis.

Keywords: Glucosamine sulphate, Hyaluronic acid, Rat, Osteoarthritis
\end{abstract}

\section{INTRODUCTION}

Osteoarthritis $(\mathrm{OA})$ is a noninflammatory chronic degenerative disease characterized primarily by cartilage destruction, osteophyte formation, and subchondral sclerosis, which progressively degenerate the joints [1,2]. Cartilage diseases are highly prevalant diseases with important socioeconomic problems that cause job loss and adversely affect patient comfort all over the world. There are many treatment options currently available for OA [3-5]. Chondroprotective agents such as hyaluronic acid (HA) and glycosaminoglycans are currently used in patients with OA. However, none of the treatment methods applied is sufficient to completely cure the disease. The common goal of all applications is to prevent the pathological process leading to degenerative arthritis after cartilaginous trauma, rescue the joint from arthrosis, and to create a regeneration tissue capable of fulfilling the functions of hyaline cartilage in damaged chondral areas. HA and glucosamine sulfate (GS) are drugs that are used for a long time for the treatment of OA. There are many clinical publications about the efficacy of these drugs [2-6,7-11]. Intra-articular HA application is preferred by most physicians because it shows more promising results in patients $[2,3,12]$.

Hyaluronic acid (a glycosaminoglycan) found naturally in synovial fluid and cartilage matrix, synthesized and secreted into joints by synovial cells, fibroblasts, and chondrocytes. HA increases the viscosity and elastic nature of the synovial fluid, allowing fluid to act as a lubricant and shock absorber [13].

It has also been reported that a layer of 1-2 mm thickness on the cartilage surface contains HA [14]. Thus, HA is considered to protect the cartilage surface and soft tissues from trauma in the joints [14]. GS is a commonly used oral preparation for the treatment of OA [12]. It is a pharmacokinetic drug with positive and mild anti-inflammatory effects on cartilage and chondrocytes, depending on its pharmacological and metabolic activities $[12,15]$. Recently, GS has been reported to suppress the catabolic effects of pro-inflammatory molecules such as IL-1, which increase cartilage destruction in the treatment of OA[6]. There are insufficient knowledge and clinical experience on the effectiveness of intra-articular use of GS.

How to cite this article: Karaduman OZ, Istemi Y, Bas GE, Solak K, Midi A. Chondroprotective efficiencies of intra-articular treatment of glucosamine sulfate and hyaluronic acid. Marmara Med J 2019; 32: 112-117. doi:10.5472/marumj.637180 
Despite its controversial use, meta-analyses of clinical trials suggest that, intra-articular HA can be used in the treatment of OA. Due to this controversial situation, more work is needed and it is thought that future work will illuminate this issue [16]. This study was planned because of the widespread use of intra-articular HA despite the fact that it is controversial in the literature. Histopathologically, the effect of GS and HA on the cartilage in OA rat model were examined.

\section{MATERIALS and METHODS}

This study was approved by the Institutional Animal Experiments Local Ethics Committee of Düzce University, School of Medicine (number, 2010/05, date 18.02.2010). The study was performed as described in the literature [5]. A total of 45 adult male Wistar Albino rats weighing 220-275 grams and 5-7 months old were used in the study. Rats were divided into 3 groups as 15 rats in each group. The first group was GS group, 2nd group was HA group and the 3rd group was control group. During the experiment, rats were housed in 3-4 rats in each cage, fed with standard laboratory nutrients, without liquid and nutrient restriction.

One rat from the GS group, three rats from the HA group and four rats from the control group died in the experimental stage, also three rats from the control group were excluded from the study because of the obvious infection findings. A total of 34 specimens (14 rats from the GS group, 12 rats from the HA group and 8 rats from the control group) were prepared for histopathological examination.

\section{Anterior Cruciate Ligament Transection Procedure}

Animals were sedated before surgery with an intraperitoneal injection of ketamine $(75 \mathrm{mg} / \mathrm{kg})$ and metomidine $(0.5 \mathrm{mg} /$ $\mathrm{kg})$. Subcutaneous buprenorphine $(0.03 \mathrm{mg} / \mathrm{kg})$ was given immediately after surgery and twice daily for 3 days to provide postoperative analgesia.

The rats were placed in supine position and draped in a sterile manner. A longitudinal incision was made in the knees of the rats starting from the upper part of the patella and extending to the tubercle of the tibia. Arthrotomy was then performed with medial parapatellar approach. The anterior cruciate ligament was macroscopically tilted to the patella laterally and excised with the aim of creating an experimental OA model [5] (Figure 1A). Complete anterior cruciate ligament (ACL) transection was confirmed by manual test for anterior laxity of the joint. The peripatellar capsular incision was then closed using 4-0 Vicryl sutures (Ethicon, Somerville, New Jersey). After the excision, the front drawer test was performed and the operation was verified.

After the surgery, no fixation was applied to the knees of the rats and free circulation was allowed. Two weeks after the surgery, intra-articular injections were performed once a week for 3 weeks, with one-week intervals to each group. In group 1 (GS group) $12 \mathrm{mg} / 0.06$ cc GS (Dona ampul ${ }^{\oplus}$, Abdi İbrahim, Istanbul, Turkey) was injected intra-articularly with an insulin injector. In

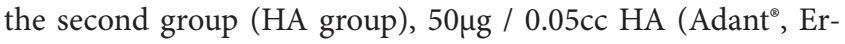
Kim drug) and in the third group (control group), $50 \mu \mathrm{l} / 0.05 \mathrm{cc}$
$0.9 \%$ sodium chloride $(\mathrm{NaCl})$ were applied. Rats were sacrificed by high dose ketamine (Ketalar ${ }^{\oplus}$, Pfizer) and specimens were evaluated histopathologically after 8 weeks of surgery and 4 weeks after injection. Arthrotomy was performed on the lower right extremities of the rats. Distal femur and tuberosity were obtained by osteotomy from the tibial side (Figure 2).

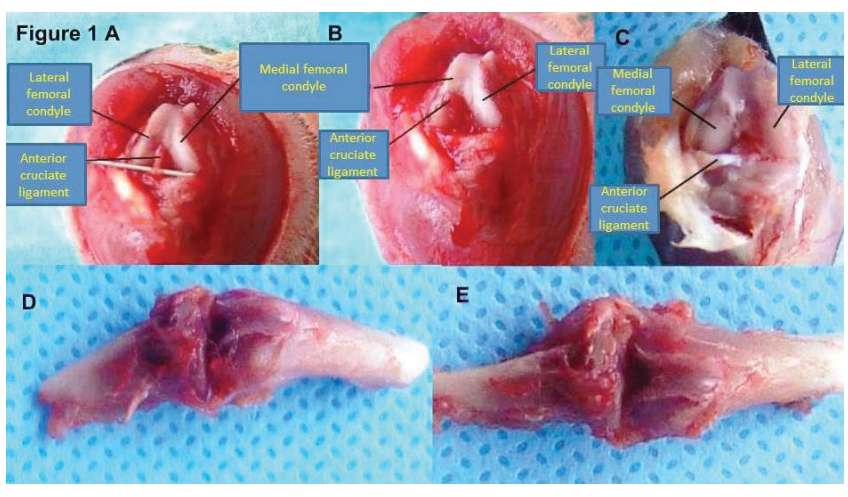

Figure 1. A: Arthrotomy performed rat knee joint, B: Rat knee joint with anterior cross ligation, C: Macroscopic image of the specimen obtained from the GS group eight weeks after the surgery. D: Macroscopic appearance of the specimen obtained from the HA group eight weeks after the surgery. E: Macroscopic appearance of the specimen obtained from the control group eight weeks after the surgery.

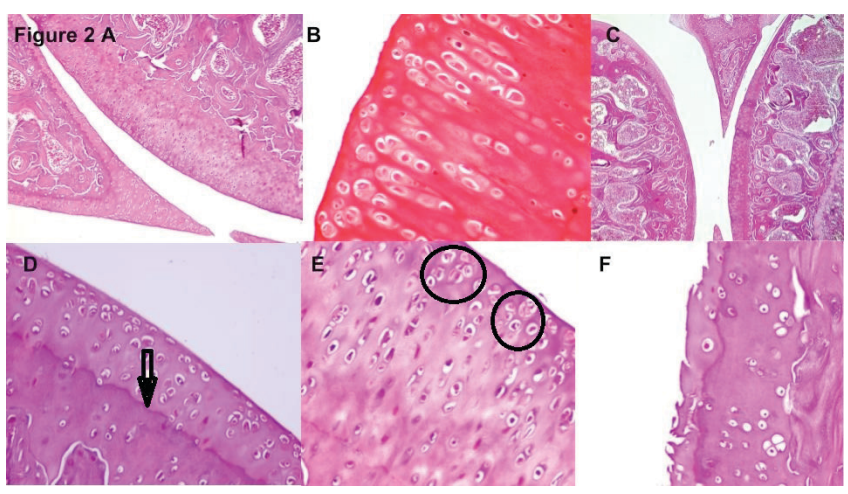

Figure 2. A: Normal articular surface, stable knee (H\&E x40), B: Loss of total staining in cartilage matrix with safranin $\mathrm{O}$ dye in the control group. (Safranin-O x400), C: Normal articular surface in the GS group (H\&E x100), D: Tidemark intact in the GS group. The continuity of tidemark is indicated by an arrow. (H\&E x100), E: Cell cloning in the HA group is shown by circles (H\&E x400), F: Tidemark intact in HA group (H\&E x100).

\section{Intra-articular Injections}

Before we performed the intra-articular injections, anesthesia was induced in a chamber with $5 \%$ isoflurane. Once anesthetized, the animal was placed in supine position with its nose set in a cone and dosed with $2 \%$ isoflurane to maintain anesthesia. The knee was shaved and prepared to provide a sterile field. Intraarticular injections were then performed through the patellar tendon with the knee in flexion using a $3 / 10 \mathrm{~mL}, 29$-gauge 
pediatric insulin syringe. Intra-articular placement of the injection was confirmed by the lack of resistance to flow with injection and by feeling the distention of the knee joint capsule, medial to the patellar tendon.

\section{Histopathological Evaluation}

Samples were randomly numbered and sent to pathology. Materials were fixed in 10\% formaldehyde for one week. The tissues were decalcified for 5 days (Shandon TBD-2) following fixation. After decalcification treatment, tissues were divided into two sections, which were perpendicular to the joint space from the joint of the medial condyle and blocked. Samples were labeled according to the number, sent to the laboratory without knowing which group they belonged to. These samples were washed in the stream water for 3 hours to remove the decalcification solution and the tissues were fixed for 2 more days in formaldehyde. Later, tissues processed for 13 hours in an automatic tissue processor (Shandon Excelsior ES, Thermo Scientific, Runcorn, England). In this procedure, formaldehyde 2 times for 30 minutes, alcohol 6 times for 60 minutes, xylene 3 times for 60 minutes, and once, 60 minutes and twice, 80 minutes paraffin were applied to the tissues. Hematoxylin \& eosin (H\&E) and safranin-O staining were performed by taking 2 micron thick sections from the paraffin-embedded tissues. The outcome measurement was a blind assessment without knowing the groups. The sections were evaluated under a light microscope (Olympus Bx-50, Olympus Optical). The findings were evaluated according to the Mankin Scale [10]. According to the Mankin Scale, preparations were evaluated in terms of cartilage structure, cellular abnormality, matrix staining and tidemark integrity (Table I).

Table I. Mankin Scale [10]

\begin{tabular}{|l|l|}
\hline I. Structure & 0 \\
\hline a. Normal & 1 \\
\hline b. Surface irregularities & 2 \\
\hline c. Pannus and surface irregularities & 3 \\
\hline d. Clefts to transitional zone & 4 \\
\hline e. Clefts to radial zone & 5 \\
\hline f. Clefts to calcified zone & 6 \\
\hline g. Complete disorganisation & \multicolumn{2}{|l|}{} \\
\hline II. Cells & 0 \\
\hline a. Normal & 1 \\
\hline b. Diffuse hypercellularity & 2 \\
\hline c. Cloning & 3 \\
\hline d. Hypocellularity & \multicolumn{2}{|l|}{} \\
\hline III. Safranin-O staining & 0 \\
\hline a. Normal & 1 \\
\hline b. Slight reduction & 2 \\
\hline c. Moderate reduction & 3 \\
\hline d. Severe reduction & 4 \\
\hline e. No dye noted & \multicolumn{2}{|l}{} \\
\hline IV. Tidemark integrity & 0 \\
\hline a. Intact & 1 \\
\hline b. Destructed & \\
\hline
\end{tabular}

In the structural evaluation, surface irregularities, pannus formation, clefts to transitional zone, clefts to radial zone, clefts to calcified zone, complete disorganization parameters were evaluated and scored. (Table I). In the cellular evaluation, diffuse hypercellularity, cloning, and hypocellularity parameters were evaluated. In the safranin-O dye, it was evaluated whether there was a decrease in matrix staining with safranin-O. In the tidemark integrity evaluation, it was evaluated whether there was a tidemark destructed or not.

It was defined as surface irregularities when there was mild tissue loss on the joint surface (Figure 3B). Grading was performed according to the depth of the existing cleft (cleft reaching transitional zone, radial zone, and the calcified zone) (Figure 3A). Complete loss of the cartilage layer was evaluated as complete disorganization (Figure 3E). When evaluating cellularity, the highest (bad) score was given to decrease cellularity. Group formation of chondrocytes in the cartilage layer was evaluated as cloning (Figure 2E).

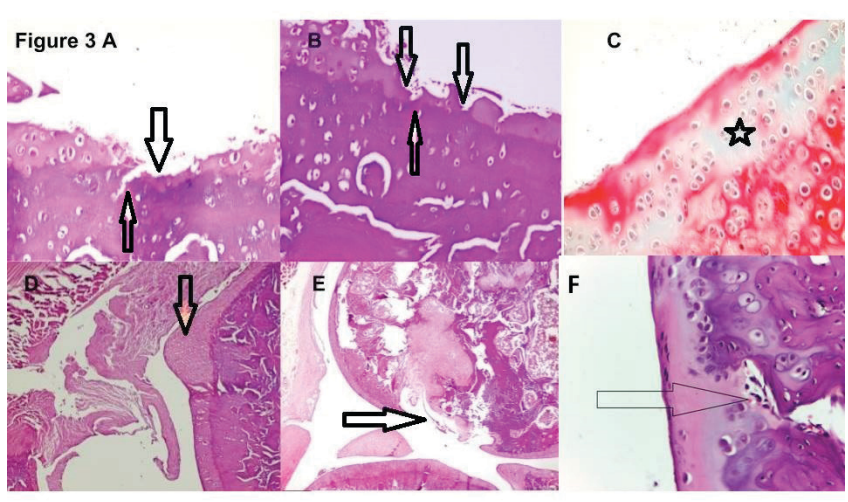

Figure 3. A; Surface irregularities (arrow pointing downwards), Cleft extending the calcified zone (arrow pointing upwards) in HA group ( $\mathrm{H} \& \mathrm{E}$ $\mathrm{x} 200$ ), B: Loss of superficial cartilage layer in HA group is indicated by an arrow (H\&E x200), C: Positive matrix stainin in GS group with safranin $\mathrm{O}$ indicated by a star (Safranin-O x400), D: Loss of the cartilage layer on the surface and pannus formation indicated by an arrow (H\&E x100), E: Complete surface cartilage layer loss in the control group (H\&E x40), F: Tidemark destruction in the control group (H\&E x100).

\section{Statistical analysis}

Histological evaluation data were recorded and statistical analyses were performed using SPSS 20.0 (SPSS, Chicago, Illinois). The distribution of continuous variables was evaluated by the one-sample Kolmogorov-Smirnov test. Kruskal-Wallis test and Mann-Whitney-U test were used in the intergroup comparisons because the distribution of the relevant variables was not normal. A p value of less than 0.05 was considered statistically significant.

\section{RESULTS}

Total Mankin Scale scores were 2.64 $\pm 2.56,3.58 \pm 3.9,8.12 \pm 2.80$ for GS group, HA group and control group respectively. 
The values of the total Mankin Scale, cartilage structure, cellular abnormality, matrix staining and tidemark integrity of the GS and HA groups were lower than values of the control group. These differences were statistically significant.

The values of the total Mankin Scale, cartilage structure, cellular abnormality, matrix staining and tidemark integrity of the GS group were lower than the values of the HA group but this difference was not statistically significant.

The mean results, minimum and maximum values of the histological examinations of the groups according to the Mankin Scale are given in table II.

During the histopathological examinations, the photographs of the preparations were taken (Figure 1-3).

Table II. The mean results, minimum and maximum values of the histological examinations of the groups according to the Mankin Scale.

\begin{tabular}{|c|c|c|c|c|c|c|}
\hline & $\begin{array}{l}\text { Structure } \\
\text { of } \\
\text { cartilage }\end{array}$ & $\begin{array}{l}\text { Cellular } \\
\text { abnorma- } \\
\text { lity }\end{array}$ & $\begin{array}{l}\text { Matrix } \\
\text { painting }\end{array}$ & $\begin{array}{l}\text { Tidemark } \\
\text { integrity }\end{array}$ & $\begin{array}{l}\text { Total } \\
\text { Mankin } \\
\text { Scale }\end{array}$ & $\mathbf{p}$ \\
\hline $\begin{array}{l}\text { GS } \\
\text { group }\end{array}$ & $\begin{array}{l}1.71 \pm 1.44 \\
(0-5)\end{array}$ & $\begin{array}{l}0.36 \pm 0.50 \\
(0-1)\end{array}$ & $\begin{array}{l}0.36 \pm 0.63 \\
(0-2)\end{array}$ & $\begin{array}{l}0.21 \pm 0.43 \\
(0-1)\end{array}$ & $2.64 \pm 2.56$ & \multirow{3}{*}{$\begin{array}{l}\mathrm{p}>0.05 \\
(\mathrm{GS}, \mathrm{HA}) \\
\mathrm{p}<0.01 \\
\text { (GS } \\
\text { Control, } \\
\text { HA } \\
\text { control) }\end{array}$} \\
\hline $\begin{array}{l}\text { HA } \\
\text { group }\end{array}$ & \begin{tabular}{|l|}
$1.83 \pm 2.04$ \\
$(0-6)$ \\
\end{tabular} & \begin{tabular}{|l|}
$0.66 \pm 0.98$ \\
$(0-3)$ \\
\end{tabular} & $\begin{array}{l}0.75 \pm 0.97 \\
(0-3)\end{array}$ & \begin{tabular}{|l|}
$0.25 \pm 0.45$ \\
$(0-1)$ \\
\end{tabular} & 3.94 & \\
\hline $\begin{array}{l}\text { Control } \\
\text { group }\end{array}$ & $\begin{array}{l}3.5 \pm 1.60 \\
(2-6)\end{array}$ & $\begin{array}{l}1.88 \pm 0.64 \\
(1-3)\end{array}$ & $\begin{array}{l}1.88 \pm 0.64 \\
(1-3)\end{array}$ & \begin{tabular}{|l}
$0.88 \pm 0.35$ \\
$(0-1)$ \\
\end{tabular} & $8.12 \pm 2.80$ & \\
\hline
\end{tabular}

GS: Glucosamine sulphate, HA: Hyaluronic acid

\section{DISCUSSION}

In this study, the chondroprotective efficacy of GS and HA was compared in an experimental osteoarthritis model. The results for GS and HA groups were better than the control group. The values of the total Mankin Scale, cartilage structure, cellular abnormality, matrix staining and tidemark integrity of the GS group were lower than the values of the HA group.

Although, the histopathological effects of the GS application when compared with the HA application were quantitatively different in favor of GS, the chondroprotective effect was not statistically significant. According to this result, intra-articular use of GS was not more effective than HA, but, we can suggest that GS might also be effectively used like HA for OA.

Many conservative and surgical approaches to prevent or slow down the OA process, or to treat the osteoarthritic joint, are currently underway [17]. Research is still underway for the ideal procedure. Recently, disease-modifying agents have been emphasized $[4,7,8]$. Several studies have been conducted on HA and have been reported in many studies that have modified OA. In the literature, $\mathrm{HA}$ is routinely used in the treatment of joint $\mathrm{OA}$. There are very few studies in the literature comparing the effects of intra-articular administration of GS and HA $[4,8,18]$. In some studies, it has been shown that GS use is more beneficial than HA use $[4,7,8]$.
Özkan et al. have compared the chondroprotective efficacy of $\mathrm{N}$-acetyl GS and HA in the treatment of early-stage OA [4]. In this study, 32 New Zealand rabbits were used and OA was formed by cutting the anterior cruciate ligaments. The animals were divided into 4 groups and 2 weeks after the operation, to the first group intra-articular N-acetyl GS, to the second group intra-articular HA, to the third group intra-articular HA and intra-muscular glucosamine and to the fourth group (control group) isotonic solution were given. Eight weeks later the rabbits were sacrificed. Macroscopically, except the control group, the other groups maintained the cartilage surface, microscopically, in groups one,two and three chondroprotective effect was demonstrated but there was no significant difference between these groups in terms of chondroprotective efficacy. In our study, GS was found to be more effective than HA in terms of chondroprotective efficacy but these results were not statistically significant.

In one study, the clinical use of GS was shown to be more beneficial than nonsteroidal anti-inflammatory agent (NSAID) use [9]. In this study, patients were compared with nonsteroidal anti-inflammatory drugs for the symptomatic effect of glucosamine in 200 patients with OA. Patients were divided into two groups. Group 1 was given daily oral $1500 \mathrm{mg}$ GS and the another group was given $1200 \mathrm{mg}$ ibuprofen. Similar positive results were achieved (GS 48\%, ibuprofen 52\%). Side effects were $35 \%$ in the ibuprofen group and $6 \%$ in the GS group.

The use of HA injections is controversial but may be considered in selected patients including those with less advanced knee OA, refractory to other nonoperative treatment options and poor surgical candidate patients $<65$ years old [19].

There are different recommendations on the use of HA injections from various professional organizations. American Academy of Orthopaedic Surgeons (AAOS) cannot recommend the use of intra-articular HA for patients with symptomatic OA of the knee, based on lack of efficacy with a low likelihood of achieving clinically important benefits [20,21].

Osteoarthritis Research Society International (OARSI) states use of intra-articular HA injections is of uncertain appropriateness in patients with knee-only OA with or without relevant comorbidities and is not appropriate in patients with multi-joint OA with or without relevant comorbidities [22].

According to some clinical researches, the intra-articular HA injection may modestly improve function at 6 months in adults $\geq$ 65 years old with knee OA. Comparing HA injection to placebo injection, $\mathrm{HA}$ is associated with small but significantly improved function at 6-month follow-up in the analysis of 10 trials [23].

Bannuru et al., compared intra-articular administration of HA to intra-articular placebo. At 3 months, intra-articular HA was associated with significant improvement in pain, function, and stiffness [16].

In addition, there are some researches that intra-articular HA injection may increase the risk of serious adverse events in adults with knee OA [24]. In another study, it was determined that these side effects were not very important [23]. 
Studies in the literature have generally achieved better results in groups using HA and GS molecules than in control groups although there are few reports showing opposite results [4,8]. GS has been used in different medical studies and the results have been reported to be most effective in these studies. Although, there are many studies in the literature on oral GS application, there are limited studies on the intra-articular application $[4,8]$. In our study, it was demonstrated that GS has positive effects on the experimental OA model in terms of histopathologic control and chondroprotective compared to HA groups. In our opinion, GS can be used intra-articularly, but this should be supported by more studies.

\section{Conclusion}

Although, the literature appears to support the use of intraarticular HA injections for the treatment of knee OA, according to our results GS can also be used intra-articularly.

In the future, the intra-articular use of GS may become an effective method of treatment for knee joint injuries and OA and studies will continue to be helpful to determine the most appropriate utilization in clinical practice.

\section{REFERENCES}

[1] Nelson AE, Allen KD, Golightly YM, Goode AP, Jordan JM. A systematic review of recommendations and guidelines for the management of osteoarthritis: The chronic osteoarthritis management initiative of the U.S. bone and joint initiative. Semin Arthritis Rheum 2014 ;43:701-12. doi: 10.1016/j. semarthrit.2013.11.012.

[2] Bannuru R R, Vaysbrot E E, Sullivan M C, McAlindon T E. Relative efficacy of hyaluronic acid in comparison with NSAIDs for knee osteoarthritis: a systematic review and metaanalysis. Semin Arthritis Rheum 2014;43:593-9. doi:10.1016/j. semarthrit.2013.10.002.

[3] Sen C, Güneş T, Saygi B, Erdem M, Köseoğlu R D, Kilic N. The chondroprotective effect of intra-articular hyaluronic acid at early stages of osteoarthritis: An experimental study in rabbits. Acta Orthop Traumatol Turc 2004;38:348-52.

[4] Özkan ÜF, Özkan K, Ramadan S, Güven Z. Chondroprotective effect of $\mathrm{N}$-acetylglucosamine and hyaluronate in early stages of osteoarthritis an experimental study in rabbits. Bulletin of the NYU Hospital for Joint Diseases 2009; 67:352-7.

[5] Orak M. M, Ak D, Midi A, et al. Comparison of the effects of chronic intra-articular administration of tenoxicam, diclofenac, and methylprednisolone in healthy rats. Acta Orthop Traumatol Turc 2015;49:438-46. doi: 10.3944/ AOTT.2015.14.0312.

[6] Herrero-Beaumont G, Rovati LC, Castañeda S, AlvarezSoria MA, Largo R. The reverse glucosamine sulfate pathway: application in knee osteoarthritis. Expert Opin Pharmacother. 2007 Feb;8(2):215-25. DOI: 10.1517/14656566.8.2.215

[7] Cen X, Liu Y, Wang S, Yang X, Shi Z, Liang X et al. Glucosamine oral administration as an adjunct to hyaluronic acid injection in treating temporomandibular joint osteoarthritis. Oral Dis. 2018 Apr;24(3):404-411. doi: 10.1111/odi.12760.

[8] Torrent A, Montell E, Vergés J, et al. SAT0547 Chondroprotective activity of acti-joint ${ }^{\oplus}$, a combination of chondroitin sulphate, glucosamine and a natural ingredient rich in hyaluronic acid. Ann Rheum Dis 2014;73(Suppl 2):789-9. doi: 10.1016/j.joca.2014.02.602

[9] Müller-Fassbender H, Bach GL, Haase W, Rovati LC, Setnikar I. Glucosamine sulfate compared to ibuprofen in osteoarthritis of the knee. Osteoarthritis Cartilage 1994; 2:61-9.

[10] van der Sluijs JA, Geesink RG, van der Linden AJ, Bulstra SK, Kuyer R, Drukker J. The reliability of the Mankin score for osteoarthritis. J Orthop Res 1992, 10:58-61. doi:10.1002/ jor.110.010.0107

[11] Heybeli N, Doral M N, Atay O A, Leblebicioglu G, Uzumcugil A. Intra-articular sodium hyaluronate injections after arthroscopic debridement for osteoarthritis of the knee: a prospective, randomized, controlled study. Acta Orthop Traumatol Turc 2008;42:221-7.

[12] Şükür E, Talu C, Akman Y E, Çirci E, Öztürkmen Y, Tüzüner T. Comparison of the chondroprotective effect of a novel hydrogel compound and traditional hyaluronate on rat cartilage in a papain-induced osteoarthritis model. Acta Orthop Traumatol Turc 2016;50:458-63. doi: 10.1016/j.aott.2016.07.008

[13] Ayhan E, Kesmezacar H, Akgun I. Intraarticular injections (corticosteroid, hyaluronic acid, platelet rich plasma) for the knee osteoarthritis. World J Orthop 2014;5:351-61. doi: 10.5312/wjo.v5.i3.351

[14] Akman Y E, Sukur E, Senel A, Sukur N E O, Talu C K, Ozturkmen Y. The comparison of the effects of a novel hydrogel compound and traditional hyaluronate following micro-fracture procedure in a rat full-thickness chondral defect model. Acta Orthop Traumatol Turc 2017;51:331-336. doi: 10.1016/j.aott.2017.04.001.

[15] Reginster JY, Deroisy R, Rovati LC, et al. Long-term effects of glucosamine sulphate on osteoarthritis progression: A randomised, placebo-controlled clinical trial. Lancet 2001; 357:251-6.

[16] Bannuru R R, Schmid CH, Kent DM, Vaysbrot EE, Wong J B, McAlindon TE. Comparative effectiveness of pharmacologic interventions for knee osteoarthritis: a systematic review and network meta-analysis.Ann Intern Med 2015;162:46-54. doi: 10.7326/M14-1231.

[17] Murat N, Karadam B, Ozkal S, Karatosun V, Gidener S. Quantification of papain-induced rat osteoarthritis in relation to time with the Mankin score. Acta Orthop Traumatol Turc 2007; 41:233-7.

[18] Cen X, Liu Y, Wang S, Yang X, Shi Z, Liang X. Glucosamine oral administration as an adjunct to hyaluronic acid injection in treating temporomandibular joint osteoarthritis. Oral Dis 2018 ;24:404-411. doi: 10.1111/odi.12760. Epub 2017 Oct 4.

[19] Jones B. Q, Covey C. J, SINEATH M. H, Sineath Jr, M. H. Nonsurgical Management of Knee Pain in Adults. Am Fam Physician 2015;92:875-83. 
[20] Hochberg MC, Altman RD, April KT, Benkhalti M, Guyatt G, McGowan J at al. American College of Rheumatology 2012 recommendations for the use of nonpharmacologic and pharmacologic therapies in osteoarthritis of the hand, hip, and knee. Arthritis Care Res (Hoboken). 2012 ;64:465-74.

[21] American Academy of Orthopaedic Surgeons (AAOS). Treatment of osteoarthritis of the knee. Evidence-based guideline. 2nd edition. AAOS 2013 May 18 PDF, summary can be found at AAOS 2013 May 18 PDF.

[22] McAlindon TE, Bannuru RR, Sullivan MC, et al. OARSI guidelines for the non-surgical management of knee osteoarthritis. Osteoarthritis Cartilage 2014;22:363-88. doi: 10.1016/j.joca.2014.01.003.
[23] O'Hanlon CE, Newberry SJ, Booth M, et al. Hyaluronic acid injection therapy for osteoarthritis of the knee: concordant efficacy and conflicting serious adverse events in two systematic reviews. Systematic reviews $2016 ; 5: 186$.

[24] Rutjes AW, Jüni P, da Costa BR, Trelle S, Nüesch E, Reichenbach S. Viscosupplementation for osteoarthritis of the knee: a systematic review and meta-analysis. Ann Intern Med 2012;157:180-91. doi: 10.7326/0003-4819-157-3201208.070.00473.

[25] Fibel KH, Hillstrom HJ, Halpern BC. State-of-the-Art management of knee osteoarthritis. World J Clin Cases 2015 ;3:89-101. doi: 10.12998/wjcc.v3.i2.89. 